\title{
Treating Hepatobiliary Cancer: The Immunologic Approach
}

\author{
A.G. Duffy T.F. Greten \\ Gastrointestinal Malignancies Section, Thoracic-GI Oncology Branch, Center for Cancer Research, National Cancer \\ Institute, National Institutes of Health, Bethesda, MD, USA
}

\section{Keywords}

Immune - Inflammation - Immune-evasion · Hepatobiliary · Cholangiocarcinoma

\begin{abstract}
Hepatobiliary cancer comprises a heterogeneous group of malignancies in which the standard treatments for advanced disease are minimally effective and evolve slowly over time. Like the majority of gastrointestinal cancers, with some notable exceptions, the impact of immune-based approaches is yet to be experienced. Notwithstanding this, the etiological background of hepatobiliary cancer - overlapping in almost every known causative or associated factor with inflammation - provides a strong clue that these approaches may have an impact on this group of diseases. This review seeks to put the management of hepatobiliary cancers in the context of its inflammation-based etiology, with the aim of pointing to the therapeutic opportunities in immune-based approaches currently entering the clinic or those that are about to do so.

(c) 2017 S. Karger AG, Basel
\end{abstract}

\section{Introduction}

The term hepatobiliary cancer refers to primary malignancies, which develop in the liver, either hepatocellular carcinoma (HCC), or the intra- and extra-hepatic biliary ductal system giving rise to biliary tract carcinomas (BTC). Hepatobiliary cancers have in common trends in global prevalence where striking peaks in incidence in certain regional hotspots contrast with relative rarity in western countries. For example, gallbladder cancer is a leading cause of cancer mortality in Chile but has an incidence of approximately in 5,000 people in the United States [1]. The practical consequence of this is that standard of care evolves slowly with a general paucity of randomized studies. For advanced metastatic disease, the modest standard of care comprises either sorafenib (for HCC) or gemcitabine-based chemotherapy (for BTC), based on the so-called SHARP and $\mathrm{ABC}-02$ trials respectively $[2,3]$. Beyond that there is no outright standard second-line option and clearly there is an unmet need here to improve the treatment options at every stage for patients with this group of malignancies.

\section{KARGER}

(c) 2017 S. Karger AG, Basel

E-Mail karger@karger.com

www.karger.com/ddi
Austin G. Duffy

Gastrointestinal Malignancies Section, Thoracic-GI Oncology Branch, Center for Cancer Research, National Cancer Institute, National Institutes of Health, 9000 Rockville Pike, 10/12N224, Bethesda, MD 20892 (USA)

E-Mail duffya@ mail.nih.gov 
The past number of years have seen much progress for immune-based approaches in solid tumor malignancies, with FDA approvals for various strategies, including dendritic cell vaccination as well as so-called immune checkpoint inhibition [4-6]. The next frontier is for these treatments to prove themselves in diseases that have thus far proved refractory. So far, with notable exceptions, this has included cancers of the gastrointestinal tract, including hepatobiliary cancers as a group. One of the first studies evaluating PD1/programmed death ligand (PD-L1)directed therapy was disappointing from a GI cancer viewpoint [7]. Similarly negative results for GI cancers were seen in other studies of checkpoint inhibition when used as a single modality [8-10].

\section{Inflammation and Predisposition to Hepatobiliary Cancer}

The causative link between inflammation and cancer development stretches back to the 19th-century observations of Rudolph Virchow. Chronic inflammation can greatly facilitate cancer development through a number of means, culminating in an immunosuppressed microenvironment containing dysfunctional "exhausted" $\mathrm{T}$ cells, high expression of inhibitory receptors (e.g., CTLA4) and cytokine milieu weighted towards suppression versus activation $[11,12]$. This broad and sometimes paradoxical relationship between cancer and inflammation is particularly relevant in hepatobiliary cancers, a group of malignancies whose predominantly associated predisposing conditions have chronic inflammation as their common underlying pathological denominator (Fig. 1). The dominant known risk factor for HCC is cirrhosis induced by long-standing inflammation from a variety of causes - viral hepatitis, environmental factors such as alcohol, metabolic syndromes and, more appreciably, non-alcoholic steatohepatitis. Similarly, for biliary tract cancers gallbladder carcinoma - the most common subtype of BTC in the United States - cholelithiasis-induced chronic inflammation, whereas - worldwide - infestation of the biliary ducts by parasites or persistent hepatolithiasis are important predisposing factors for intrahepatic cholangiocarcinoma (ICC) [13]. In the United States and Europe, primary biliary cirrhosis and sclerosing cholangitis are all established risk factors for BTC, as are causes of intrahepatic inflammation, for example, obesity [14]. Recent studies have linked liver disease associated with the same increasing incidence of chronic hepatitis $\mathrm{C}$ virus infection, which explains much of the increase in HCC rates with ICC [15].

Treating Hepatobiliary Cancer:

The Immunologic Approach

\section{Immunogenicity of Hepatobiliary Cancer}

The acknowledgement of an immune role in the etiology of hepatobiliary cancer is important because it raises the possibility of an immune-based remedy where the predisposition and pathogenesis can be manipulated for therapeutic gains. The success of immune checkpoint inhibition is dependent upon the presence of an existing endogenous anti-tumor immune response. Spontaneous immune responses including T-cell responses [16] as well as humoral responses to different tumor-associated antigens [17] have been described in HCC. A number of studies have described that tumor infiltrating $\mathrm{CD} 4+$ regulatory T-cells correlate with poor outcome in patients who undergo surgical resection $[18,19]$. Likewise, spontaneous immune responses also occur in BTC. This was illustrated in a dramatic manner by Tran and colleagues who performed whole-exomic-sequencing on the resected tumor of a patient with metastatic chemo-refractory cholangiocarcinoma [20]. By generating tandem minigene constructs of each of the somatic tumor mutations and co-culturing with expanded populations of isolated tumor infiltrating lymphocytes (TIL), they identified a reactive population of CD4+ cells, an enriched population of which the investigators were able to adoptively transfer to produce tumor regression. It has been postulated that the degree to which these anti-tumor responses exist is related to the mutagenic burden of the tumor. Hepatobiliary cancers in general are not highly mutated. In HCC, the median number of mutations is 39 [21]. The number is similarly low in BTC. Whole-exome sequencing of 239 cases of BTC revealed median numbers of mutations across the ICC, extrahepatic cholangiocarcinoma and gallbladder cancer subtypes of 39, 35, and 64 [22]. This relatively average degree of mutagenicity in the setting of spontaneous anti-tumor immunity challenges somewhat the direct relationship between mutagenicity and immunogenicity, which is clearly relevant but not absolute. Indeed, Tran and colleagues have recently suggested that this relationship is more nuanced, with the finding of immunoreactive TIL even in the setting of low mutational burden [23].

Perhaps the best indicator of an existing antitumor immunity is the presence of TIL. In HCC, the presence of TIL has been shown to be prognostic [24, 25]. In BTC, the prevalence and prognostic relevance of TIL have also been documented. Sabbatino et al. [26] evaluated lymphocyte infiltration in 27 cases of ICC. All tumors had TIL, and this correlated with HLA expression. Nakakubo et al. [27] investigated the significance of TIL pres- 


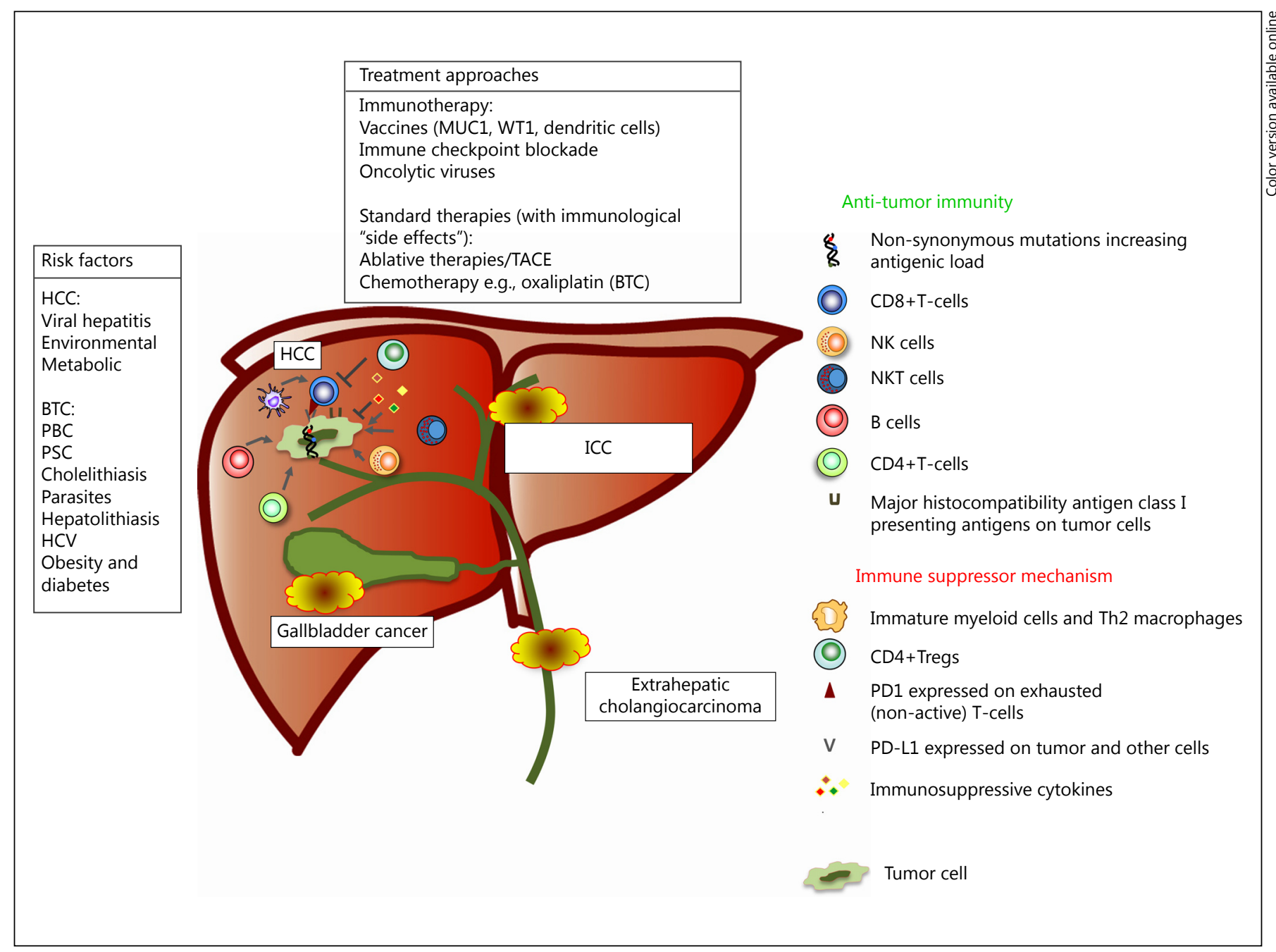

Fig. 1. Immunological events described in hepatobiliary cancer: risk factors, immune-based approaches, immune-activating and immunosuppressive events.

ence in 110 resected gallbladder specimens and found that both $\mathrm{CD} 4+$ and CD8+ T cell infiltration correlated with decreasing tumour invasion and survival. Goeppert et al. [28] evaluated immune cell infiltration in 375 cases of BTC following resection. Approximately half the number of patients had some degree of TIL and this was also found to correlate with survival. Interestingly, patients who had higher total regulatory $\mathrm{T}$ lymphocyte counts had a better survival outcome. The prognostic value of Tregs in other GI tumor types has been varying in terms of whether it was a favorable or adverse factor [29].

Another method of immune evasion by tumors is achieved by changes in the expression downregulation of MHC I [30-32]. HCCs express low levels of MHC class I molecules in addition to costimulatory molecules [33]. Similarly, in BTC, Goeppert et al. [34] analysed the impact of MHC expression on survival in a large series of patients $(n=334)$ as well as the relationship of this to TIL. MHC I expression was assessed semi-quantitatively and divided into 2 groups of high and low expression. There was a trend for higher MHC I expression in lower disease stages and an association between MHC I expression and the number of TIL. Interestingly, the investigators found that associations differed based on the BTC subtype, with the prognostic significance of MHC I expression, being by trend stronger in extrahepatic disease compared with intrahepatic disease, suggesting that mechanisms of immune escape may vary with anatomical subtype. 
In addition to evasive mechanisms, tumors have the ability to suppress antitumor immunity in a variety of ways. Myeloid-derived suppressor cells, a heterogeneous cell population that suppress T-cell responses, have been shown to accumulate in the blood of patients with hepatobiliary malignancies $[35,36]$. A separate mechanism results from the expression by tumors of PD-L1, which also inhibits T-cell reactivity. PD-L1 is heavily expressed in HCC and has been shown to be of prognostic significance $[37,38]$. Similarly, in BTC, Ye et al. [39] evaluated the expression of PD-L1 and its ligand PD- 1 in $n=31$ surgically resected cases in addition to the corresponding cancer-adjacent tissues. Expression of PD-L1 was found to be upregulated in cholangiocarcinoma tissues compared with the cancer-adjacent tissues. Tumor-related PD-L1 expression was significantly correlated with both tumor differentiation and pTNM stage and was inversely correlated with $\mathrm{CD} 8+$ tumor-infiltrating lymphocytes. In the analysis by Sabbatino et al. [26] 8 of 27 ICC cases expressed PD-L1.

\section{Translational Immune-Based Approaches in Hepatobiliary Cancers}

Immunotherapy has been tested in hepatobiliary cancers for many years, particularly HCC. Most studies in the past have either used cytokine-based or antigen-based approaches [35]. While these studies have generally proven to be safe with induction - in peripheral blood or serum - of tumor-specific immune responses, a majority have failed to show that this translates into clinical efficacy. In more recent times, however, evidence of efficacy has been demonstrated in a number of immune modalities, mainly in HCC.

\section{Hepatocellular Carcinoma}

The use of immune-based approaches is especially attractive for HCC, given the invariable underlying existence of cirrhosis and related problems such as thromocytopenia. The development of conventional cytotoxic regimens, particularly those requiring hepatic metabolism, is therefore an additional challenge in this cancer over others. The most promising immune-based approaches tested in HCC have been oncolytic viruses and immune checkpoint inhibitors. The use of oncolytic virus as a treatment strategy in HCC is a recent development. JX-594 is an oncolytic poxvirus modified by the

Treating Hepatobiliary Cancer:

The Immunologic Approach insertion of $\beta$-galactosidase, a surrogate marker for the detection of viral gene expression and human GM-CSF to stimulate anti-tumor immune responses. The vaccinia virus replicates in cancer cells dependent on cellular thymidine kinase activity [40]. Intra-tumoral application of JX-594 was shown to be safe in a phase I study in HCC [41]. Results from a second study in 30 patients with advanced HCC were subsequently reported in which patients were randomized for treatment with low-dose $\left(10^{8} \mathrm{PFU}\right)$ or high-dose ( $\left.10^{9} \mathrm{PFU}\right) \mathrm{JX}-594$ administered by intra-tumoral injection [42]. Objective responses rates were $15 \%$ in both groups and an intrahepatic disease control of $50 \%$ was achieved. It should be noted that disease control rates were equivalent in injected and distant untreated tumors suggesting the presence of a systemic immune response. There was a clear dose relationship with regard to overall survival (6.7 months in the low and 14.1 months in the high dose). Immune-monitoring studies demonstrated that at least 11 of 16 patients developed HCC-specific antibody responses as determined by the analysis of complement-dependent cytotoxicity of patients' serum against HCC cell lines. Cellular immune responses were studied by ELISpot analysis against $\beta$-gal and they demonstrated the induction of $\beta$-gal specific T cells, which were found in one patient 18 months after treatment.

The other strategy that has shown promise in HCC recently is immune checkpoint inhibition. Tremelimum$\mathrm{ab}$ - a fully human monoclonal antibody that binds to CTLA-4 expressed on the surface of activated T lymphocytes and results in inhibition of B7-CTLA-4-mediated downregulation of T-cell activation - was initially tested in a pilot clinical trial in 20 patients with advanced HCC and HCV infection [43]. Tremelimumab, at a dose of $15 \mathrm{mg} / \mathrm{kg}$ every 90 days i.v., was tolerated well and showed evidence of activity. Three out of 17 evaluable patients developed confirmed partial responses and 10 patients had stable disease as the best response to treatment. HCVspecific T-cell responses were studies conducted in these patients as a surrogate marker for the anti-CTLA4-induced immune responses. Recently, preliminary results for a representative agent of the other most prominent class of immune checkpoint inhibitors currently in clinic, PD1 blockade, were presented [44]. In this study, $n=47$ patients with preserved liver function but advanced HCC were treated with nivolumab, with impressive, preliminary evidence of activity. Out of 39 evaluable patients, there were 2 complete responses and 7 partial responses.

One of the unique features of HCC is the reliance upon interventional radiologic procedures in everyday 
patient management, either for the purposes of curative intent (heat-based or cryo-ablation) or disease control (TACE). Systemic therapy plays a comparatively modest role. Following ablation, tumor antigens from necrotic tumor are taken up by antigen-presenting cells (mainly DCs), resulting in activating tumor-specific immune responses $[45,46]$. Ablated tumor tissue has been shown to promote DC maturation $[47,48]$. A tumor-specific immune response may prevent recurrent disease in addition to treating distant metastases. Several preclinical and clinical studies have documented an increase in peripheral immunity following ablation [47, 49-52]. We are currently conducting a study evaluating subtotal RFA in combination with anti-CTLA4 therapy (NCT01853618) in patients with HCC and BTC. Provisionally, this approach is feasible and safe, with some early evidence of efficacy, which needs to be confirmed with more number of patients and more mature followup [53].

\section{Biliary Tract Carcinomas}

In terms of published clinical data for immune approaches in BTC, the experience is dominated by peptide vaccines. These small studies have been well tolerated without showing a strong signal of efficacy $[54,55]$. For example, Aruga et al. [56] conducted a small phase I clinical trial evaluating multiple-peptide vaccination for patients with advanced BTC. Peptide-specific T-cell immune responses and disease stability were observed in some patients. Dendritic cell-based vaccines have also been evaluated, most commonly in combination with standard modalities, which complicates assessment of efficacy $[57,58]$. The experience with checkpoint inhibitors is very preliminary. Documented response to antiCTLA-4 therapy has been observed, manifesting as a delayed response after initial disease progression [59]. The early data for anti-PD1 inhibition has been recently presented, showing encouraging evidence of efficacy, and which seems consistent with use of this agent in other solid tumor studies [60].

One of the advantages of immune-based approaches is the potential role of their combination with standard therapies - cytotoxic agents, radiation or interventional radiologic procedures [61]. Certain chemotherapeutics can activate rather than suppress the immune system and a robust immune response is a necessary component determining tumor response [62]. Gemcitabine is a nucleoside analogue that is part of the standard treat- ment, and probably the most common chemotherapeutic agent used in BTC $[3,63]$. The immune effects of gemcitabine have been studied perhaps more than for any other drug used in GI cancer. Its effects on the immune system are diverse [61]. With regard to BTC, Koido et al. [64] demonstrated that in ICC cells isolated from a patient with malignant ascites, immunogenic modulation of the cells could be induced by gemcitabine with the upregulation of MHC class I and II, calreticulin - a modulator or immunogenic cell death - MUC1 and WT1 mRNA [64]. Interestingly, the authors found that gemcitabine also induced the upregulation of immunosuppressive $\mathrm{PD}-\mathrm{L} 1$, suggesting a potential rationale for combination therapy.

\section{Conclusions}

The advantages of immune-based treatments are clear, such that the initial results of the checkpoint inhibitors in particular have captured the imaginations of physicians and the general public alike. While the benefit seen thus far is real, hepatobiliary malignancies have not been among the banner group of malignancies to have so far shown dramatic and durable evidence of efficacy. This is beginning to change with the presentation of what so far is a relatively small volume of clinical experience, albeit encouraging. The close etiological relationship between hepatobiliary cancer and inflammation speaks to an important role for the immune system in these diseases, as do the data discussed showing by almost any measure - TIL, MHC downregulation, PD-L1 expression - evidence of a reactive surveillance, which can be potentially manipulated by the new drugs and technologies that are making their way in the clinic.

\section{Disclosure Statement}

The authors report no financial conflicts of interest to declare.

\section{Financial Support}

This research was supported by the Intramural Research Program of the NIH, National Cancer Institute, Center for Cancer Research. The content of this publication does not necessarily reflect the views or policies of the Department of Health and Human Services, nor does mention of trade names, commercial products, or organizations imply endorsement by the US Government. 


\section{References}

1 Nogueira L, Foerster C, Groopman J, et al: Association of aflatoxin with gallbladder cancer in Chile. JAMA 2015;313:2075-2077.

2 Llovet JM, Ricci S, Mazzaferro V, et al: Sorafenib in advanced hepatocellular carcinoma. N Engl J Med 2008;359:378-390.

3 Valle J, Wasan H, Palmer DH, et al: Cisplatin plus gemcitabine versus gemcitabine for biliary tract cancer. N Engl J Med 2010;362: 1273-1281.

4 Kantoff PW, Higano CS, Shore ND, et al: Sipuleucel-T immunotherapy for castration-resistant prostate cancer. N Engl J Med 2010; 363:411-422.

5 Hodi FS, O'Day SJ, McDermott DF, et al: Improved survival with ipilimumab in patients with metastatic melanoma. N Engl J Med 2010;363:711-223.

6 Robert C, Thomas L, Bondarenko I, et al: Ipilimumab plus dacarbazine for previously untreated metastatic melanoma. N Engl J Med 2011;364:2517-2526.

7 Brahmer JR, Tykodi SS, Chow LQ, et al: Safety and activity of anti-PD-L1 antibody in patients with advanced cancer. N Engl J Med 2012;366:2455-2465.

8 Topalian SL, Hodi FS, Brahmer JR, et al: Safety, activity, and immune correlates of antiPD-1 antibody in cancer. N Engl J Med 2012; 366:2443-2454.

9 Chung KY, Gore I, Fong L, et al: Phase II study of the anti-cytotoxic T-lymphocyte-associated antigen 4 monoclonal antibody, tremelimumab, in patients with refractory metastatic colorectal cancer. J Clin Oncol 2010;28:34853490.

10 Royal RE, Levy C, Turner K, et al: Phase 2 trial of single agent Ipilimumab (anti-CTLA-4) for locally advanced or metastatic pancreatic adenocarcinoma. J Immunother 2010;33: 828-833.

11 Jiang Y, Li Y, Zhu B: T-cell exhaustion in the tumor microenvironment. Cell Death Dis 2015;6:e1792.

12 Trinchieri G: Cancer and inflammation: an old intuition with rapidly evolving new concepts. Annu Rev Immunol 2012;30:677706.

13 Rizvi S, Gores GJ: Pathogenesis, diagnosis, and management of cholangiocarcinoma. Gastroenterology 2013;145:1215-1229.

14 Chang JS, Tsai CR, Chen LT: Medical risk factors associated with cholangiocarcinoma in Taiwan: a population-based case-control study. PLoS One 2013;8:e69981.

15 El-Serag HB, Engels EA, Landgren $\mathrm{O}$, et al: Risk of hepatobiliary and pancreatic cancers after hepatitis $C$ virus infection: a populationbased study of U.S. veterans. Hepatology 2009; $49: 116-123$

16 Mizukoshi E, Nakamoto Y, Arai K, et al: Comparative analysis of various tumor-associated antigen-specific t-cell responses in patients with hepatocellular carcinoma. Hepatology 2011;53:1206-1216.
17 Korangy F, Ormandy LA, Bleck JS, et al: Spontaneous tumor-specific humoral and cellular immune responses to NY-ESO-1 in hepatocellular carcinoma. Clin Cancer Res 2004;10:4332-4341.

$18 \mathrm{Fu} \mathrm{J}, \mathrm{Xu} \mathrm{D}$, Liu Z, et al: Increased regulatory $\mathrm{T}$ cells correlate with CD8 T-cell impairment and poor survival in hepatocellular carcinoma patients. Gastroenterology 2007; 132: 2328-2339.

19 Gao Q, Qiu SJ, Fan J, et al: Intratumoral balance of regulatory and cytotoxic $\mathrm{T}$ cells is associated with prognosis of hepatocellular carcinoma after resection. J Clin Oncol 2007;25 2586-2593.

20 Tran E, Turcotte S, Gros A, et al: Cancer immunotherapy based on mutation-specific $\mathrm{CD} 4+\mathrm{T}$ cells in a patient with epithelial cancer. Science 2014;344:641-645.

21 Vogelstein B, Papadopoulos N, Velculescu VE, Zhou S, Diaz LA Jr, Kinzler KW: Cancer genome landscapes. Science 2013;339:15461558 .

22 Nakamura H, Arai Y, Totoki Y, et al: Genomic spectra of biliary tract cancer. Nat Genet 2015;47:1003-1010.

23 Tran E, Ahmadzadeh M, Lu YC, et al: Immunogenicity of somatic mutations in human gastrointestinal cancers. Science 2015;350: 1387-1390.

24 Garnelo M, Tan A, Her Z, et al: Interaction between tumour-infiltrating $\mathrm{B}$ cells and $\mathrm{T}$ cells controls the progression of hepatocellular carcinoma. Gut 2017;66:342-351.

25 Chew V, Tow C, Teo M, et al: Inflammatory tumour microenvironment is associated with superior survival in hepatocellular carcinoma patients. J Hepatol 2010;52:370379.

26 Sabbatino F, Villani V, Yearley JH, et al: PDL1 and HLA class I antigen expression and clinical course of the disease in intrahepatic cholangiocarcinoma. Clin Cancer Res 2016; 22:470-478.

27 Nakakubo Y, Miyamoto M, Cho Y, et al: Clinical significance of immune cell infiltration within gallbladder cancer. Br J Cancer 2003; 89:1736-1742.

28 Goeppert B, Frauenschuh L, Zucknick M, et al: Prognostic impact of tumour-infiltrating immune cells on biliary tract cancer. Br J Cancer 2013;109:2665-2674.

29 Zhuo C, Xu Y, Ying M, et al: FOXP3+ Tregs: heterogeneous phenotypes and conflicting impacts on survival outcomes in patients with colorectal cancer. Immunol Res 2015;61:338347.

30 Campoli M, Ferrone S: HLA antigen changes in malignant cells: epigenetic mechanisms and biologic significance. Oncogene 2008;27: 5869-5885.

31 Campoli M, Ferrone S: Tumor escape mechanisms: potential role of soluble HLA antigens and NK cells activating ligands. Tissue Antigens 2008;72:321-334.
32 Ryschich E, Nötzel T, Hinz U, et al: Control of T-cell-mediated immune response by HLA class I in human pancreatic carcinoma. Clin Cancer Res 2005;11(2 pt 1):498-504.

33 Fujiwara K, Higashi T, Nouso K, et al: Decreased expression of B7 costimulatory molecules and major histocompatibility complex class-I in human hepatocellular carcinoma. J Gastroenterol Hepatol 2004;19: 1121-1127.

34 Goeppert B, Frauenschuh L, Zucknick M, et al: Major histocompatibility complex class I expression impacts on patient survival and type and density of immune cells in biliary tract cancer. Br J Cancer 2015;113:1343-1349.

35 Greten TF, Duffy AG, Korangy F: Hepatocellular carcinoma from an immunologic perspective. Clin Cancer Res 2013;19:66786685.

$36 \mathrm{Xu}$ XD, Hu J, Wang M, et al: Circulating myeloid-derived suppressor cells in patients with pancreatic cancer. Hepatobiliary Pancreat Dis Int 2016;15:99-105.

37 Gao Q, Wang XY, Qiu SJ, et al: Overexpression of PD-L1 significantly associates with tumor aggressiveness and postoperative recurrence in human hepatocellular carcinoma. Clin Cancer Res 2009;15:971-979.

38 Zeng Z, Shi F, Zhou L, et al: Upregulation of circulating PD-L1/PD-1 is associated with poor post-cryoablation prognosis in patients with HBV-related hepatocellular carcinoma. PLoS One 2011;6:e23621.

39 Ye Y, Zhou L, Xie X, Jiang G, Xie H, Zheng S: Interaction of $\mathrm{B} 7-\mathrm{H} 1$ on intrahepatic cholangiocarcinoma cells with PD-1 on tumor-infiltrating T cells as a mechanism of immune evasion. J Surg Oncol 2009;100:500-504.

40 Kirn DH, Thorne SH: Targeted and armed oncolytic poxviruses: a novel multi-mechanistic therapeutic class for cancer. Nat Rev Cancer 2009;9:64-71.

41 Park BH, Hwang T, Liu TC, et al: Use of a targeted oncolytic poxvirus, JX-594, in patients with refractory primary or metastatic liver cancer: a phase I trial. Lancet Oncol 2008;9: 533-542.

42 Heo J, Reid T, Ruo L, et al: Randomized dosefinding clinical trial of oncolytic immunotherapeutic vaccinia JX-594 in liver cancer. Nat Med 2013;19:329-336.

43 Sangro B, Gomez-Martin C, de la Mata M, et al: A clinical trial of CTLA-4 blockade with tremelimumab in patients with hepatocellular carcinoma and chronic hepatitis C. J Hepatol 2013;59:81-88.

44 El-Khoueiry AB, Melero I, Crocenzi TS, et al: Phase I/II safety and antitumor activity of nivolumab in patients with advanced hepatocellular carcinoma (HCC): CA209-040. J Clin Oncol 2015;33:LBA101.

45 Greten TF, Korangy F: Radiofrequency ablation for the treatment of HCC-maybe much more than simple tumor destruction? J Hepatol 2010;53:775-776.
Treating Hepatobiliary Cancer:

The Immunologic Approach 
46 den Brok MH, Sutmuller RP, Nierkens S, et al: Efficient loading of dendritic cells following cryo and radiofrequency ablation in combination with immune modulation induces anti-tumour immunity. Br J Cancer 2006;95: 896-905.

47 Zerbini A, Pilli M, Penna A, et al: Radiofrequency thermal ablation of hepatocellular carcinoma liver nodules can activate and enhance tumor-specific T-cell responses. Cancer Res 2006;66:1139-1146.

48 Zerbini A, Pilli M, Fagnoni F, et al: Increased immunostimulatory activity conferred to antigen-presenting cells by exposure to antigen extract from hepatocellular carcinoma after radiofrequency thermal ablation. J Immunother 2008;31:271-282.

49 Hansler J, Wissniowski TT, Schuppan D, et al: Activation and dramatically increased cytolytic activity of tumor specific T lymphocytes after radio-frequency ablation in patients with hepatocellular carcinoma and colorectal liver metastases. World J Gastroenterol 2006; 12:3716-3721.

50 Hiroishi K, Eguchi J, Baba T, et al: Strong CD8(+) T-cell responses against tumor-associated antigens prolong the recurrence-free interval after tumor treatment in patients with hepatocellular carcinoma. J Gastroenterol 2010;45:451-458.

51 Johnson EE, Yamane BH, Buhtoiarov IN, et al: Radiofrequency ablation combined with KS-IL2 immunocytokine (EMD 273066) results in an enhanced antitumor effect against murine colon adenocarcinoma. Clin Cancer Res 2009;15:4875-4884.

52 Nijkamp MW, Borren A, Govaert KM, et al: Radiofrequency ablation of colorectal liver metastases induces an inflammatory response in distant hepatic metastases but not in local accelerated outgrowth. J Surg Oncol 2010; 101:551-556

53 Duffy AG, Makarova-Rusher OV, Kerkar SP, et al: A pilot study of tremelimumab - a monoclonal antibody against CTLA-4 - in combination with either trans catheter arterial chemoembolization (TACE) or radiofrequency ablation (RFA) in patients with hepatocellular carcinoma (HCC). J Clin Oncol 2015;33:4081.

54 Kaida M, Morita-Hoshi Y, Soeda A, et al: Phase 1 trial of Wilms tumor 1 (WT1) peptide vaccine and gemcitabine combination therapy in patients with advanced pancreatic or biliary tract cancer. J Immunother 2011;34:92-99.

55 Yamamoto K, Ueno T, Kawaoka T, et al: MUC1 peptide vaccination in patients with advanced pancreas or biliary tract cancer. Anticancer Res 2005;25:3575-3579.

56 Aruga A, Takeshita N, Kotera Y, et al: Phase I clinical trial of multiple-peptide vaccination for patients with advanced biliary tract cancer. J Transl Med 2014;12:61.

57 Kobayashi M, Sakabe T, Abe H, et al: Dendritic cell-based immunotherapy targeting synthesized peptides for advanced biliary tract cancer. J Gastrointest Surg 2013;17: 1609-1617.
58 Lepisto AJ, Moser AJ, Zeh H, et al: A phase I/II study of a MUC1 peptide pulsed autologous dendritic cell vaccine as adjuvant therapy in patients with resected pancreatic and biliary tumors. Cancer Ther 2008;6:955964.

59 Shimomura A, Fujiwara Y, Kondo S, et al: Tremelimumab-associated tumor regression following after initial progression: two case reports. Immunotherapy 2016;8:9-15.

60 Bang YJ, Doi T, De Braud F, Piha-Paul S, Hollebecque A, Abdul Razak AR, Lin CC, Ott PA, He AR, Yuan SS, Koshiji M, Lam B, Aggarwal R: Safety and efficacy of pembrolizumab (MK-3475) in patients (pts) with advanced biliary tract cancer: Interim results of KEYNOTE-028. Eur J Cancer 2015;51(suppl 3):S112.

61 Duffy AG, Greten TF: Immunological off-target effects of standard treatments in gastrointestinal cancers. Ann Oncol 2014;25:24-32.

62 Liu WM, Fowler DW, Smith P, Dalgleish AG: Pre-treatment with chemotherapy can enhance the antigenicity and immunogenicity of tumours by promoting adaptive immune responses. Br J Cancer 2010;102:115-123.

63 Burris H, Storniolo AM: Assessing clinical benefit in the treatment of pancreas cancer: gemcitabine compared to 5-fluorouracil. Eur J Cancer 1997;33(suppl 1):S18-S22.

64 Koido S, Kan S, Yoshida K, et al: Immunogenic modulation of cholangiocarcinoma cells by chemoimmunotherapy. Anticancer Res 2014;34:6353-6361. 